Relations industrielles

Industrial Relations

\title{
Organisation professionnelle : réalisations étrangères
}

\section{Émile Gosselin}

Volume 15, numéro 4, octobre 1960

URI : https://id.erudit.org/iderudit/1021944ar

DOI : https://doi.org/10.7202/1021944ar

Aller au sommaire du numéro

Éditeur(s)

Département des relations industrielles de l'Université Laval

ISSN

0034-379X (imprimé)

1703-8138 (numérique)

Découvrir la revue

Citer ce document

Gosselin, É. (1960). Organisation professionnelle : réalisations étrangères.

Relations industrielles / Industrial Relations, 15(4), 489-492.

https://doi.org/10.7202/1021944ar

Tous droits réservés @ C Département des relations industrielles de l’Université Laval, 1960
Ce document est protégé par la loi sur le droit d'auteur. L'utilisation des services d'Érudit (y compris la reproduction) est assujettie à sa politique d'utilisation que vous pouvez consulter en ligne.

https://apropos.erudit.org/fr/usagers/politique-dutilisation/ 
Pour atteindre un tel objectif, deux choses sont nécessaires: 1) la constitution chez les différents groupes organisés, de cadres dirigeants et de conseillers, non seulement sincères et bien intentionnés, mais aussi très bien équipés en fait de connaissances techniques relativement aux problèmes qui sont leurs, ayant la confiance de leurs ressortissants et aptes à incarner les idéologies dans des solutions pratiques, concrètement réalisables;

2) le relèvement du niveau général d'éducation chez les membres de ces groupes, syndicats, associations patronales, etc., en sorte que le contrôle démocratique des décisions chez eux soit exercé de façon efficace et prévienne les abus du pouvoir sous toutes ses formes.

Il est essentiel aussi, pour que la collaboration soit possible et réelle, qu'un certain équilibre des pouvoirs soit sauvegardé à la fois entre les groupes en présence et entre ces groupes réunis et le pouvoir de l'Etat. Il faut donc à tout prix, non seulement prévenir ou éliminer, selon le cas, les excès de pouvoirs d'un ou de plusieurs groupes vis-à-vis des autres, mais veiller à ce que la somme des pouvoirs privés ne dépasse à aucun prix ceux de la communauté incarnés par les pouvoirs publics, l'Etat. L'élaboration de concert avec l'Etat, des modalités du contrôle nécessaire à cet effet, est à coup sûr, un terrain de choix où la collaboration des intéressés est en mesure de s'exercer.

\section{ORGANISATION PROFESSIONNELLE: RÉALISATIONS ÉTRANGÈRES}

\section{Entile Gosselin}

Les modes de collaboration entre patrons et travailleurs s'encadrent dans des structures et adoptent une multiplicité de formes qui varieront selon les réalités propres au pays ou au secteur de production où ils s'établissent. Ils peuvent atteindre différents degrés de perfectionnement selon les endroits.

En certains pays, la conjoncture économique ou politique, ou encore la faiblesse des groupes organisés, appellent un degré d'intervention étatique très poussé dans les rapports patronaux-ouvriers. En d'autres endroits, des facteurs culturels ou idéologiques, ou la maturité des organisations patronales et ouvrières, permettent aux groupes organisés une très grande autonomie dans l'établissement des rapports de collaboration.

\section{Hollande}

Au lendemain de la guerre, les syndicats patronaux et ouvriers décidèrent de mettre en oeuvre les plans de reconstruction qu'ils avaient élaborés dans la clan. destinité du maquis. Ils optèrent pour une étroite collaboration avec le gouvernement dans la poursuite des objectifs nationaux. Les confédérations patronales et ouvrières mirent sur pied un organisme bipartite, le Front national, que le gouvernement considère comme la voie officielle du monde patronal et ouvrier en matière économique et sociale. Tout est maintenant une stricte indépendance politique, cet organisme constitue l'un des principaux conseillers du gouvernement. 
Le Front national joue un rôle prépondérant dans la négociation des conventions collectives nationales et dans l'établissement des taux de salaires nationaux. La Commission des Conciliateurs gouvernementaux et le Ministre des Affaires Sociales ne ratifieront aucune décision en ces diverses matières sans avoir auparavant soumis ces projets à l'examen et à la critique du Front national. L'action de cet organisme s'appuie, au niveau du secteur de production et de l'entreprise, sur des Conseils de Métier, organisme bipartite, qui cherchent à réconcilier les points de vue différents. L'établissement de politique à longue portée en matière économique et politique est, depuis 1949, du ressort du Conseil Socio-économique, un organisme tripartite dont le champ d'action est beaucoup plus vaste que celui du Front national lequel s'intéresse surtout aux relations patronales-ouvrières.

La centralisation très poussée en Hollande dans l'aménagement des rapports patronaux-ouvriers aurait pu facilement conduire à la bureaucratisation et même à l'immobilisme. Mais la multiplication des Conseils de Métiers a donné une force nouvelle aux groupements locaux et favorisé les négociations au palier de l'entreprise et du secteur professionnel, faisant ainsi contrepoids à une trop grande force concentrée au palier des confédérations. La présence du Conseil économique aux côtés de la Commission des Conciliateurs et du Front national du Travail diminue les dangers d'une trop grande centralisation, car elle permet au public de s'exprimer librement.

\section{SCANDINAvie}

Tout comme en Hollande, les grandes centrales syndicales patronales et ouvrières ont assumé, comme étant les leurs propres, les objectifs nationaux de reconstruction d'après-guerre. Sauf au Danemark, les centrales exercent un grand contrôle sur l'activité de leurs groupements affiliés. On ne négocie d'habitude que des conventions collectives nationales qui doivent recevoir la sanction des centrales patronales et ouvrières. La centrale patronale ou ouvrière exerce un contrôle rigoureux des arrêts de travail et, en cas de conflit légitime, prend elle-même l'initiative de toutes les activités en vue d'un règlement. Si un règlement s'avère impossible au niveau des centrales, le différend est soumis à des médiateurs gouvernementaux qui, d'habitude, s'abstiendront d'intervenir si les parties ne consentent pas à reprendre la production ou à la continuer. Par contre, le médiateur ne rendra pas publiques ses conclusions, si l'une des parties refuse de les accepter.

Les pays scandinaves, sauf la Norvège en certains cas, se refusent à Yarbitrage obligatoire des salaires. Le règlement des conflits d'intérêts est donc du seul ressort des centrales syndicales, car ces dernières, contrairement à ce qui se produit en Hollande, se refusent à toute intervention du gouvernement, sauf aux bons offices des médiateurs. Les conflits de droit sont du ressort de tribunaux du travail, organismes tripartites, constitués à part égale de représentants des syndicats patronaux et ouvriers et de ceux du public. Seuls les organismes reconnus peuvent comparaître devant ces tribunaux. L'arbitrage privé est à l'honneur en Scandinavie; aussi le rôle de ces tribunaux est de décider uniquement de cas ayant une véritable portée nationale.

Les centrales syndicales scandinaves se tiennent beaucoup plus à distance des gouvernements que celles de la Hollande. Sauf en Suède, l'unité syndicale est 
réalisée depuis très longtemps en Scandinavie et les centrales syndicales sont sorties moins meurtries de la guerre qu'en Hollande. Les scissions idéologiques au sein du monde patronal et ouvrier en Hollande ont obligé les divers mouvements hollandais à se donner des super-structures comme instruments de cohésion, ce qui constituait une véritable invitation aux gouvernements à jouer le rôle d'arbitre interprofessionnel. L'unité syndicale en pays scandinaves et l'absence de graves conflits idéologiques ont permis aux patrons et travailleurs de régler entre eux leurs propres problèmes, sans intervention gouvernementale, tout en coopérant avec l'Etat à la poursuite des objectifs nationaux.

\section{Grande-Bretagne}

La croissance du syndicalisme en Grande-Bretagne constitue un processus spontané, continu, ne répondant à aucun schème théorique, indépendant de l'action étatique mais toujours en relations très étroite avec les besoins qu'il doit satisfaire. Contrairement à ce qui se produit en Hollande et en Scandinavie, la centrale syndicale n'intervient pas dans la négociation des conventions collectives. Ce rôle est joué par les syndicats affiliés. Mais les négociations se font cependant sur le plan national et lient tous les employeurs et employés reliés au secteur de négociation. Les conventions d'entreprise peuvent, à l'occasion, complèter les dispositions contenues dans la convention nationale. Les syndicats anglais rejettent toute intervention gouvernementale dans les relations patronales-ouvrières en temps de paix. Mais patrons et ouvriers dans les différents secteurs ont institué des comités conjoints nationaux dont la décision est exécutoire en vue de déterminer les taux de salaires et certaines conditions de travail. L'action de ces comités se prolonge par la constitution de comités conjoints au niveau local ou régional. Ainsi, le rapport de Mr. J.H. Whitley publié en 1917 trouve finalement une application universelle.

\section{Allemagne}

Tout comme en Hollande et en Scandinavie, l'aménagement des relations patronales-ouvrières en Allemagne est soumis à une forte centralisation. Le désir des syndicats de négocier des conventions collectives nationales a favorisé la création de puissantes associations de patrons qui négocient au nom de tous leurs membres. Mais de part et d'autre, l'on préfère s'en tenir à une règlementation privée des rapports patronaux-ouvriers plutôt que de s'en remettre à l'intervention de l'Etat. L'arbitrage n'existe que du consentement des parties. Les tribunaux du travail sont des organismes tripartites dont les décisions sont d'habitude unanimes. Plutôt que d'imposer des décisions, les arbitres préfèrent concilier les parties.

Les syndicats ouvriers ont reçu de l'Etat, le droit à la co-détermination dans les aciéries et les charbonnages. Dans les autres secteurs, des conseils d'usine ont été établis. Ces organismes bipartites jouent un rôle considérable au niveau des entreprises en matière de bien-être et d'application de la convention collective. 


\section{Conclusion}

Il n'y a pas en soi de modes de collaboration valables dans tous les pays et pour tous les groupes organisés. L'expérience révèle que, pour être efficace, un cadre de collaboration ne peut s'établir à partir d'un schème purement théorique. Il doit reposer sur des structures et des expériences qui rendent justice à la réalité des faits. Mème si les rapports de collaboration dans divers pays sont en vue d'objectifs qui, dans l'ensemble, s'apparentent, il n'y a nul lieu de s'étonner que chaque pays présente un visage qui lui soit propre.

\section{LE SYNDICALISME DES CADRES}

\section{JaCQues Cousineau, s.J.}

L'importance croissante de la place qu'occupent les travailleurs non manuels dans la main-d'oeuvre des pays industriels est un des aspects les plus significatifs de l'histoire économique et sociale du XXe siècle. Cet accroissement du nombre des travailleurs non manuels est à la fois absolu et relatif. Il résulte des besoins créés par le développement économique et social; de par le monde il y a une aspiration profonde à ce que tous les groupes sociaux aient des possibilités plus grandes d'épanouissement et un accès plus large au domaine culturel et dès lors bénéficient d'une élévation progressive des niveaux de vie.

Des enquêtes récentes faites notamment sur les répercussions de l'automation permettent de conclure que l'extraordinaire développement du progrès technique et la mise sur pied de puissants ensembles industriels ont entraîné, par suite des immenses concentrations d'hommes et de moyens matériels, la multiplication des professions de techniciens et d'ingénieurs, de mème que la croissance des fonctions d'encadrement dans l'entreprise.

\section{DÉFINITION DES CADRES}

Le terme «cadre » nous vient de France où il est employé couramment depuis une vingtaine d'années. La réalité qu'il représente ne se retrouve pas exactement dans les autres pays d'Europe ni dans l'entreprise nord-américaine, bien que la réalité décrite sous le nom de supervisor s'en approche singulièrement.

De façon générale, on entend par «cadres» le groupe des personnes qui dans une entreprise «encadrent» le personnel des ouvriers même qualifiés et des employés subalternes ou inférieurs; ils «encadrent» au sens de donner de la consistance et de l'animation à l'ensemble à cause de la part d'autorité qu'ils détiennent ou de leurs qualifications techniques ou intellectuelles.

M. Jacquin, dont la recherche sur les cadres du commerce et de l'industrie fait autorité, groupe en une définition fonctionnelle les divers éléments que son optique lui a permis d'entrevoir:

«Le cadre se caractérise essentiellement comme le salarié responsable de l'appréciation et de la mise en oeuvre de tout ou partie de la politique de l'entreprise. » 